\title{
POLÍTICA NACIONAL DE PRÁTICAS INTEGRATIVAS E COMPLEMENTARES EM SAÚDE: DISCURSO DOS ENFERMEIROS DA ATENÇÃO BÁSICA
}

\section{NATIONAL POLICY ON INTEGRATIVE AND COMPLEMENTARY HEALTH PRACTICES: DISCOURSE OF PRIMARY CARE NURSING}

\section{POLÍTICA NACIONAL DE PRÁCTICAS INTEGRADORAS Y COMPLEMENTARIAS EN SALUD: DISCURSO DE LOS ENFERMEROS DE LA ATENCIÓN PRIMARIA}

Daniele Pereira Soares ${ }^{1}$, Ariane Moreira Coelho ${ }^{2}$, Luiz Eduardo Abrantes da Silva ${ }^{3}$, Raquel de Jesus Rocha da Silva ${ }^{4}$, Camila Rolim de Figueiredo ${ }^{5}$, Marcelo Costa Fernandes ${ }^{6}$.

\section{RESUMO}

Objetivo: Analisar o discurso dos enfermeiros da Atenção Básica em relação à Política Nacional de Práticas Integrativas e Complementares de Saúde. Método: Trata-se de estudo descritivo com abordagem qualitativa, realizado com 14 enfermeiros das Estratégias de Saúde da Família. Para a coleta de dados foi utilizada uma entrevista semiestruturada, após a aprovação do Comitê de Ética e Pesquisa. Resultados: Percebeu-se o desconhecimento dos enfermeiros em relação à Política Nacional de Práticas Integrativas e Complementares de Saúde, em razão das lacunas no processo formativo e falta de educação permanente, porém os profissionais pontuaram as possíveis práticas que podem ser utilizadas no cuidado e evidenciou-se, como destaque, neste estudo, a orientação de plantas medicinais e fitoterápicos e certa confusão na diferenciação entre as mesmas. Conclusão: É necessário que exista o fortalecimento na formação acadêmica dos enfermeiros, diminuindo as lacunas existentes no aprendizado, por meio da inclusão de disciplinas e apoio da gestão, oferecendo ações de educação permanente, referentes a essas formas de cuidado no cenário da Atenção Básica.
\end{abstract}

Descritores: Terapias Complementares; Cuidados de Enfermagem; Atenção Primária à Saúde.

\section{ABSTRACT}

Objective: To analyze the discourse of Primary Care nursing regarding the National Policy on Integrative and Complementary Health Practices. Method: This is a descriptive study with a qualitative approach, which was performed with 14 nurses of the Family Health Strategies. A semi-structured interview was used to collect data, after the approval of the Research Ethics Committee. Results: Nurses were unaware of the National Policy on Integrative and Complementary Health Practices due to gaps in the training process and lack of continuing education. Nevertheless, the professionals highlighted the possible practices that could be used in care, with an emphasis being placed on the orientation of medicinal plants and herbal medicines, showing some confusion in the differentiation between them. Conclusion: There is a need to strengthen the academic training of nurses, reducing the gaps in learning, through the inclusion of subjects and management support by offering continuing education actions related to these ways of care in the Primary Care setting.

Descriptors: Complementary Therapies; Nursing Care; Primary Health Care.

\section{RESUMEN}

Objetivo: Analizar el discurso de los enfermeros de la Atención Primaria con respecto a la Política Nacional de Prácticas Integradoras y Complementarias de Salud. Método: Se trata de un estudio descriptivo con enfoque cualitativo conducido con 14 enfermeros de las Estrategias de Salud Familiar. Para la recolección de datos, se utilizó una entrevista semiestructurada, después de la aprobación del Comité de Ética e Investigación. Resultados: Se notó el desconocimiento de los enfermeros acerca de la Política Nacional de Prácticas Integradoras y Complementarias de Salud debido a lagunas en el proceso formativo y falta de educación continua, pero los profesionales señalaron las posibles prácticas que pueden ser utilizadas en la atención, haciendo hincapié en este estudio en la orientación de plantas medicinales y medicaciones fitoterapéuticas, mostrando alguna confusión en la diferenciación entre las mismas. Conclusión: Se hace necesario potenciar la formación académica de los enfermeros, disminuyendo las lagunas existentes en el aprendizaje, a través de la inclusión de asignaturas y apoyo de la gestión ofreciendo acciones de educación continua referentes a esas formas de atención en el escenario de la Atención Primaria.

Descriptores: Terapias Complementarias; Atención de Enfermería; Atención Primaria de Salud.

${ }^{1}$ Enfermeira. Graduada pela Universidade Federal de Campina Grande. ${ }^{2}$ Graduanda em Enfermagem pela Universidade Federal de Campina Grande. ${ }^{3}$ Graduando em Enfermagem pela Universidade Federal de Campina Grande. ${ }^{4}$ Graduanda em Enfermagem pela Universidade Federal de Campina Grande. ${ }^{5}$ Enfermeira. Graduada pela Universidade Federal de Campina Grande. ${ }^{6}$ Doutor em Cuidados Clínicos em Enfermagem e Saúde pela Universidade Estadual do Ceará. Docente da Universidade Federal de Campina Grande.

Como citar este artigo:

Soares DP, Coelho AM, Silva LEAS, et al. Política Nacional de práticas integrativas e complementares em saúde: Discurso dos enfermeiros da Atenção Básica. Revista de Enfermagem do Centro Oeste Mineiro. 2019;9:e3265. [Access ]; Available in: DOI: http://dx.doi.org/10.19175/recom.v9i0.3265 


\section{INTRODUÇÃO}

O cuidado de enfermagem nos serviços de saúde vem se tornando cada vez mais complexo, à medida que busca a plenitude de suas ações no que diz respeito às condições de saúde, dessa maneira, a Política Nacional de Práticas Integrativas e Complementares em Saúde (PNPIC) $^{(1)}$ busca integralizar e ampliar o cuidado em seu aspecto biopsicossocial.

A PNPIC foi aprovada e publicada, no ano de 2006, com os objetivos de atuar nos campos da promoção, manutenção e recuperação da saúde e prevenção de agravos, por meio de mecanismos naturais, apresentando visão integral e humanizada do sujeito em seu processo saúde e doença. Observa-se, ainda, com base nesta política, a promoção do cuidado sensível a partir da inclusão da participação social e do autocuidado, destacando-se a Atenção Básica (AB) como cenário para a produção dessas práticas $^{(1)}$.

A partir das portarias ministeriais, foram adicionadas uma extensão de Práticas Integrativas e Complementares em Saúde (PICS), a primeira Portaria no 971 de 03 de dezembro de 2006, trouxe diretrizes que contemplam e institucionalizam no Sistema Único de Saúde (SUS) a oferta de serviços e produtos da medicina tradicional chinesa/acupuntura, homeopatia, plantas medicinais e fitoterapia, termalismo social/crenoterapia e medicina antroposófica ${ }^{(2)}$.

Em 2017, foram adicionadas mais 14 práticas, dentre elas: arteterapia, ayurveda, biodança, dança circular, meditação, musicoterapia, naturopatia, osteopatia, quiropraxia, reflexoterapia, reiki, shantala, terapia comunitária integrativa e yoga ${ }^{(3)}$. E, no ano de 2018 , foi lançada a mais nova portaria que inclui novas práticas à PNPIC, como: aromaterapia, apiterapia, bioenergética, constelação familiar, cromoterapia, geoterapia, hipnoterapia, imposição de mãos, ozonioterapia e terapia de florais ${ }^{(4)}$.

Apesar da importância da PNPIC e de suas práticas, as mesmas ainda seguem com entraves, como a dificuldade na ampliação do acesso, na oferta e no financiamento para o fortalecimento desses serviços, sendo pouco utilizadas no cotidiano assistencial, em decorrência da precária condição de formação de recursos humanos para atuar nas PICS e a carência de recursos materiais e infraestrutura para a sua efetivação ${ }^{(5)}$.

Mesmo com o déficit no cotidiano assistencial, a utilização das práticas tem repercussões positivas, estudos confirmam que os pacientes que têm possibilidade de tratamento com as PICS, obtêm aumento em sua qualidade de vida, confirmando seu êxito nas fragilidades deixadas no campo da saúde pelo paradigma fragmentado do modelo biomédico ${ }^{(6)}$.

A profissão brasileira que se tornou pioneira no reconhecimento das práticas alternativas e complementares para ser utilizada pelos profissionais da saúde foi a enfermagem. Nessa concepção de práticas de cuidado, o profissional de enfermagem possibilita a troca e a construção de novos saberes, oportunizando a autonomia do próprio sujeito em relação a sua saúde ${ }^{(7)}$.

Logo, para o desenvolvimento deste estudo, estabeleceu-se a seguinte questão norteadora: qual o discurso dos enfermeiros da Atenção Básica em relação à Política Nacional de Práticas Integrativas e Complementares em Saúde?

Este estudo apresenta a relevância de fomentar as PICS com embasamento na política, contribuindo na formação dos profissionais e estimulando a utilização dessas práticas tanto pelo enfermeiro quanto pela comunidade, além de ser importante ferramenta na construção do vínculo entre profissional e sujeito, na participação ativa no seu processo de saúde, como também na melhoria da assistência prestada, servindo para futuras reflexões acerca dos benefícios dessas formas de cuidado no cotidiano da AB.

Dessa maneira, objetivou-se analisar o discurso dos enfermeiros da Atenção Básica em relação à Política Nacional de Práticas Integrativas e Complementares em Saúde.

\section{MÉTODO}

O estudo em questão é de natureza descritiva com abordagem qualitativa. Foi realizado na Atenção Básica do município de Cajazeiras, no estado da Paraíba. Essa cidade faz parte da 4a Macrorregião de Saúde e da 9a Gerência Regional de Saúde da Paraíba, apresentando, atualmente, 19 Unidades Básicas de Saúde (UBS), compostas por 23 Equipes de Saúde da Família cadastradas.

Os participantes deste estudo foram 14 enfermeiros que compõem as 23 Equipes de Saúde da Família do município de Cajazeiras. O critério de inclusão utilizado foi trabalhar há mais de doze meses como enfermeiro na $A B$, compreendendo-se que esse seja período 
satisfatório para estabelecer o vínculo com a dinâmica desse cenário de atenção. Foram adotados como critérios de exclusão: estar de férias, de licença-saúde ou afastado do serviço. 0 número final de participantes, isto é, 14 se deu, após a identificação da saturação teórica da coleta de dados.

A coleta de dados ocorreu entre os meses de maio e junho de 2017 e foi realizada por meio de uma entrevista individual, em local reservado na própria UBS em que o enfermeiro atuava, com duração média de 10 minutos, guiada por meio de questionário semiestruturado que continha perguntas discursivas voltadas ao conhecimento dos enfermeiros acerca da PNPIC, de suas características e da sua realização no cotidiano assistencial.

Após a coleta, ordenação e organização dos dados gerados, por meio das entrevistas semiestruturadas, foi utilizado, como processo metodológico para a análise dos dados, o Discurso do Sujeito Coletivo (DSC), que é um método que possibilita a representação do pensamento de uma determinada coletividade.

O DSC propõe a soma de ideias, de forma não numérica, que operacionalizadas metodologicamente expressem o pensamento de um determinado grupo por meio do discurso. Entende-se o DSC como um projeto de organização e tabulação de informações qualitativas de cunho verbal, obtidas de depoimentos que, basicamente, analisa o material verbal coletado para se extrair dele as Ideias-Centrais (IC) e suas correspondentes Expressões-Chaves (ECH). Esses depoimentos irão compor a matéria-prima, sob a forma de um ou vários discursos-sínteses na primeira pessoa do singular, melhor dizendo, na primeira pessoa (coletiva) do singular, onde, ao mesmo tempo em que evidencia a presença do indivíduo do discurso, faz uma referência coletiva, pois esse ser individual fala em nome de uma coletividade $^{(8)}$.

A investigação foi iniciada após a aprovação do projeto pelo Comitê de Ética em Pesquisa (CEP) da Universidade Federal de Campina Grande (UFCG), campus de Cajazeiras, sob o número do parecer 2.012.802. A participação na pesquisa se realizou mediante a assinatura do entrevistado no Termo de Consentimento Livre e Esclarecido (TCLE), elaborado em duas vias, assinado pelo participante da entrevista e pela pesquisadora responsável. Respeitando a resolução 510/2016 do Ministério da Saúde, os componentes éticos e legais estão presentes em todas as etapas da pesquisa, confirmando aos participantes sigilo e privacidade das informações que foram coletadas, assegurando-Ihes a sua utilização para fins científicos e acadêmicos.

\section{RESULTADOS E DISCUSSÃO}

Esta pesquisa foi realizada com total de 14 enfermeiros na $A B$ da Cidade de Cajazeiras, Paraíba. Em relação ao perfil dos participantes, houve predominância feminina, sendo 11 do sexo feminino e três do sexo masculino. A maioria (10) tinha mais de cinco anos de formados, porém, destaca-se que oito apresentavam menos de cinco anos de atuação na UBS. Cabe mencionar ainda que somente um dos entrevistados era graduado, os demais haviam cursado especializações, em sua maioria (oito) em saúde da família.

No que se refere à política, os enfermeiros demonstram desconhecimento da mesma, porém, ainda utilizam de algumas PICS, mesmo sem embasamento teórico. Identificaram-se três categorias principais que serão expostas e analisadas com o seu respectivo DSC. A primeira categoria apresenta o desconhecimento dos enfermeiros a respeito da política que retrata as PICS, expondo três núcleos centrais de ideias, o déficit no conhecimento da PNPIC, a ausência dessa política no processo formativo e carência de capacitações. Já a segunda categoria identifica as PICS como possibilidade do cuidado multidimensional, gerado a partir de um núcleo central, a identificação das práticas mais conhecidas e seus objetivos. A terceira categoria aborda as plantas medicinais e fitoterápicos como possibilidade de produção no cuidado cotidiano do enfermeiro, reunindo três núcleos centrais de ideias, a dificuldade em diferenciar a prática da fitoterapia e plantas medicinais, os benefícios da utilização dessa terapêutica e a diminuição de custos da população.

A primeira categoria apresenta o desconhecimento dos enfermeiros a respeito da política que aborda as PICS, foi desenvolvida a partir das entrevistas de dez enfermeiros, conforme demonstrado a seguir:

\section{Categoria 1- Desconhecimento da Política Nacional de Práticas Integrativas e Complementares em Saúde.}

DSC 01- "O que é a política em si, em si né? Não sei dizer o que é assim, eu não me recordo 
dessa política, pelo fato de não ser praticada no setor em que eu trabalho. Essa política não é implantada aqui no PSF não, porque realmente, eu desconheço o tanto de práticas que a gente poderia utilizar. Eu já escutei falar da existência dela, porém nunca li sobre a prática e essa política normalmente a gente não aplica dentro da unidade básica, eu já ouvi falar sobre a política, eu não sabia de que se tratava, mas eu tinha ouvido falar, eu nunca cheguei a fazer, não tenho capacitação para isso. É mais a falta de informação que a gente tem sobre isso, quando a gente vai pro campo de trabalho, a gente sente dificuldade de colocar em prática pelo pouco conhecimento que infelizmente ainda existe, a gente não teve como disciplina, nem teve como complementar e na prática do dia a dia a gente acaba deixando de lado essas questões. Não recebemos nunca um treinamento, também questão minha, nunca li, a política em si, o que rege ela, o que é permitido, o que não é, pra o campo de trabalho a realidade é outra, são atividades ou práticas que não estão bem no dia a dia da unidade de saúde".

Neste DSC se torna notório o déficit no que diz respeito à PNPIC, em decorrência das lacunas não preenchidas no processo formativo do enfermeiro, em seu período de graduação, bem como em razão das ausências de capacitações ofertadas pela gestão, por meio da educação permanente, o que reverbera negativamente na prática cuidativa cotidiana desse profissional, ao deixar de ofertar para a população do seu território adscrito ações que possibilitem um olhar para além da clínica tradicional, queixaconduta, prescritiva e medicamentosa.

Tal situação é comprovada no estudo sobre a situação do ensino superior das PICS nos cursos de graduação em saúde no país. Nesta pesquisa, foi demonstrado que das 87 instituições públicas de ensino superior em enfermagem analisadas, apenas 23 oferecem disciplinas relacionadas às PICS. Além disso, das poucas que as ofertam, 17 o fazem de forma optativa, isto é, a maioria dos enfermeiros está se formando sem domínio acerca da PNPIC, fazendo com que os mesmos não obtenham segurança para a implantação e implementação da mesma ${ }^{(7)}$.

Corroborando com os achados dessa investigação, um estudo realizado nas Unidades de Saúde da Família (USF) em João Pessoa, na Paraíba, evidenciou que todos os enfermeiros entrevistados, no decorrer de suas falas, demonstraram desconhecimento no que se refere à PNPIC, o que revelou ainda mais o despreparo do enfermeiro, seja na graduação, pós-graduação ou pela falta de cursos de capacitação e especialização ${ }^{(9)}$.

Outra pesquisa ocorrida em uma UBS, em São Paulo, constatou que dos 70 profissionais participantes do estudo, cerca de $56,5 \%$ desconhecem a PNPIC, e quanto à formação profissional associada às PICS, detectou-se que $68,2 \%$ dos profissionais não tiveram capacitações relacionadas ao assunto. E com relação a adquirir capacitação ou curso sobre PICS, 76,8\% negaram qualquer qualificação após início das atividades de trabalho ${ }^{(10)}$.

Desse modo, demonstra-se que depois de formados, por vezes, os profissionais ficam estagnados nas práticas convencionais e envolvem-se em ambiente de falta de embasamento científico e ausência de compreensão das políticas públicas ${ }^{(11)}$. Tal situação fomenta a diminuição da efetividade da PNPIC e de suas práticas no cenário da $A^{(12)}$.

Dessa maneira se faz necessário incorporar as PICS nas disciplinas acadêmicas, como também na educação permanente ou cursos de forma que o profissional se sinta capaz de realizar essas terapias na integralidade do cuidado ${ }^{(13-14)}$.

Mesmo sendo uma política que possui como objetivo o fortalecimento da $A B$, com ações orientadas para a prevenção de agravos e promoção e recuperação da saúde, a PNPIC, como destacado no DSC, ainda passa despercebida no cotidiano assistencial do enfermeiro. Portanto, fazem-se necessários movimentos instituintes, tanto nos espaços formadores com a inclusão de disciplinas para o despertar dos acadêmicos e docentes quanto nos próprios ambientes de trabalho com ênfase na $A B$, na busca da capacitação e sensibilização desses profissionais sobre a importância das PICS para a consolidação do cuidado mais efetivo, humanizado e ético.

A segunda categoria contou com a participação de nove enfermeiros e apresenta as PICS com base na visão dos enfermeiros:

\section{Categoria 2- Práticas Integrativas e Complementares como possibilidade do cuidado multidimensional}

DSC 02- "São práticas que ajuda o paciente a relaxar, ajuda o paciente a esquecer um pouco da dor. São bem adquiridos pelo corpo, para que tenham uma saúde mais adequada. Pode fazer uso de chás, medicação com fitoterápicos, 
calmantes, yoga, né?! Acupuntura que usa aquelas agulhas pra ativar algumas áreas do sistema nervoso, que trate, né?! Algumas doenças como estresse, problema renal, problema neurológico, é usada pra asma. Há também músicas pra ela tentar relaxar, entendeu? Que chama musicoterapia. Eu já vi também a integração em quintais, onde a comunidade fazia quintais e hortas com plantas medicinais pra que fossem usadas por eles, né?! Essa parte homeopática entre outros que, no que tange ao processo de saúde doença e as práticas do autocuidado. Elas são voltadas pra aquilo que não seja modelo curativista, potencializar essas ações é importante de forma da gente tá deixando de usar a medicalização".

Identifica-se, neste DSC, que mesmo com o desconhecimento da PNPIC, os enfermeiros apresentam a manifestação das possíveis terapêuticas que podem ser realizadas com as PICS, porém de forma bem reduzida, pois se demonstra que os enfermeiros identificam as práticas mais conhecidas e seus objetivos, indicando que a maior dificuldade é sobre o que rege a política e a busca pelas atualizações das portarias que incluem novas terapêuticas.

Ainda assim, os enfermeiros conseguem fazer a ligação que essas práticas têm com o corpo e mente e que, por vezes, mesmo de maneira tímida, expressam que o cuidado, por meio dessas, deve abranger o todo do usuário, ou seja, a singularidade e principalmente a multidimensionalidade do sujeito.

São várias as possibilidades de aplicação das PICS no cuidado, levando-se em consideração a essência do cuidado integral, porém é preciso ampliar os saberes e a divulgação sobre suas indicações, eficácia e manejo, além do investimento em pesquisas na área. Essas práticas se fazem muito úteis considerando a visão do cuidado ativo e total, visto que, complementam o tratamento convencional e prestam assistência que abrangem os aspectos físicos, psicológicos e emocionais, como também melhoram a qualidade de vida do sujeito ${ }^{(15)}$.

Analisando algumas práticas citadas pelos enfermeiros desse DSC, um estudo demonstrou o benefício da acupuntura em pacientes com lombalgia crônica, onde dos $100 \%$ que utilizaram essa prática, $40 \%$ suspenderam totalmente 0 consumo de analgésicos ${ }^{(16)}$.

Uma revisão integrativa demonstrou que a musicoterapia apresenta vários resultados positivos, dentre eles, tanto em fatores fisiológicos em relação à redução da ansiedade, dor, estresse, quadros de depressão, confusão mental, sintomas psicóticos, níveis de pressão arterial e melhor qualidade do sono, como nos fatores psicossociais como aumento da satisfação e qualidade de vida, na promoção do relaxamento e bem-estar e na melhoria das relações interpessoais ${ }^{(17)}$.

Destaca-se, ainda, como experiência exitosa, a prática do cultivo de hortas comunitárias em uma UBS, apresentou impactos positivos, demonstrando melhorias na relação profissional-usuário, além de promover o diálogo sobre autocuidado, alimentação saudável, a redução da excessiva automedicação e o incentivo de medicamentos fitoterápicos e uso de plantas medicinais ${ }^{(18)}$.

Um estudo realizado com idosos que utilizam das PICS aponta a melhoria na qualidade de vida, em relação à autoestima, alívio da dor, controle da hipertensão arterial, aumento do equilíbrio, melhora na disposição e nas relações sociais, fortalecimento das estruturas ósseas e musculares, além da diminuição do consumo de medicamentos ${ }^{(19)}$.

Logo, identifica-se uma série de benefícios das diversas exemplificações das PICS abordadas no DSC, como a fitoterapia, acupuntura, musicoterapia, entre outras, visando à qualificação do cuidado, com o fortalecimento do vínculo entre o profissional da saúde e o usuário, consolidação da relação dialogada entre os sujeitos envolvidos no plano terapêutico, bem como o protagonismo do ator social no seu autocuidado, incentivando, com isso, a reorientação do modelo de atenção à saúde para além das práticas biologistas.

Por fim, a terceira categoria aborda a utilização de plantas medicinais e fitoterápicos que, mesmo com um discurso ainda sutil, apresenta a possibilidade de cuidado no cotidiano assistencial do enfermeiro na $A B$. Para a produção do DSC dessa categoria, houve a participação de 11 enfermeiros:

Categoria 3 - Plantas medicinais e fitoterápicos como possibilidade de produção no cuidado cotidiano do enfermeiro.

DSC 03- "Eu tento fazer orientação nessa parte da fitoterapia, não na sua completude, mas focado no, o uso de ervas, né?! Seria os fitoterápicos, essa daí, acho que é mais ou menos isso. Tendo em vista que é uma terapêutica que usa plantas medicinais, né?! Essa questão do uso 
de fitoterápicos eu também acho muito útil, de lambedores, de chás. A gente orienta, por exemplo, com relação ao uso de ervas, algumas ervas a gente diz, ou algumas é, leguminosas específicas, que eu lembro muito, por exemplo, do chuchu, que eu recomendo muito aos hipertensos. A gente tá pensando em fazer uma horta medicinal, né?! A medicação de plantas medicinais, sendo feita em pacientes, poderia realmente trazer benefícios, talvez muito mais do que medicações de laboratórios. Têm várias coisas que a gente pode utilizar, que é uma maneira menos custosa como diz assim, que ia ser muito benéfica e ela não precisa de tanto material e que dê mais acesso".

Ainda que os enfermeiros apresentem desconhecimento em relação à PNPIC, nota-se que a utilização de plantas medicinais e fitoterápicos acaba emergindo no DSC, mesmo que, de forma sutil, dando destaque a essa terapêutica na possibilidade do cuidado.

São diversos os benefícios da utilização das plantas medicinais e fitoterapia como: fortalecimento do vínculo entre a comunidade e a equipe de saúde; a troca mútua de conhecimentos; a corresponsabilidade, por meio da promoção da autonomia, construção de saberes sobre as plantas medicinais geradas pela interação profissional-usuário e empoderamento do sujeito como ator social.

Outras vantagens que também são observadas com o uso de plantas medicinais e fitoterapia a partir do seu uso são: possibilidade de redução excessiva de medicalização; fortalecimento do princípio de integridade; aumento de recursos terapêuticos e ofertas de cuidados $^{(20)}$.

Outro achado é que há certa confusão no discurso dos enfermeiros, visto que ora percebese que esses profissionais são capazes de distinguir o uso de plantas medicinais e fitoterápicos e ora há momentos em que os enfermeiros não conseguem diferenciá-las, deixando parecer em seu discurso, que a forma de utilizar e orientar cada uma dessas práticas é a mesma.

O Programa Nacional de Plantas Medicinais e Fitoterápicos (PNPMF) $)^{(21)}$ estabelece essa diferença em que as plantas medicinais são espécies vegetais sob cultivo ou não, capazes de aliviar sintomas e curar doenças. Já os fitoterápicos são medicamentos industrializados que obtêm seus produtos a partir das plantas medicinais.
Corroborando com esse achado, em um estudo alguns enfermeiros mencionam a orientação e aplicação das plantas medicinais e fitoterapia, apesar do pouco conhecimento, falta de treinamento e dificuldade em compreender a fitoterapia. Essa situação aponta para a necessidade de qualificação profissional, pois a maioria relata despreparo, desde o período acadêmico, continuando esse ciclo na vida profissional, visto que a oferta de cursos de capacitações não obtém a educação necessária para esta possibilidade de cuidado ${ }^{(9)}$.

Dessa maneira, é notório o impacto que o reduzido conhecimento a respeito da utilização de plantas medicinais e fitoterápicos pode trazer. Por isso, é de suma importância que o profissional de saúde tenha conhecimento na realização das práticas com plantas medicinais e fitoterápicos para evitar o uso indevido e errôneo da população, diminuindo, dessa forma, os casos de intoxicação e maiores danos à saúde ${ }^{(22)}$. Destaca-se que um dos profissionais que poderia desmistificar o uso e tornar a população sensível à utilização dessas PICS é o enfermeiro.

Acrescentando a esse pensamento, grande parte dos profissionais não está preparada para utilizar as plantas medicinais ou prescrever fitoterápicos para a população, na sua prática cotidiana, porém essa ação pode se tornar viável e benéfica, em razão da redução de custos na $A B^{(23)}$. Fortalecendo essa ideia, outro estudo demonstra que os profissionais de saúde afirmam que as PICS com ênfase nas plantas medicinais e fitoterápicas são terapêuticas úteis no processo de cuidado, em decorrência do relativo baixo custo, o que facilita o acesso da população ${ }^{(24)}$, o que pode ser reforço positivo no plano terapêutico tradicional ao acrescentar novas e acessíveis medidas terapêuticas ${ }^{(25)}$.

Destaca-se, por fim, que na região em que foi realizada esta pesquisa, alto sertão paraibano, há uma variedade de plantas que, a partir de capacitações estruturadas na educação permanente e embasamento científico poderiam ser utilizadas na produção do cuidado, fomentando a ruptura com a prática fincada somente no tratamento medicamentoso tradicional.

\section{CONSIDERAÇÕES FINAIS}

No decorrer deste estudo, analisou-se o discurso dos enfermeiros da $A B$ em relação à PNPIC. Priorizando esse cenário de atenção, em decorrência da preferência de aplicação das PICS, 
em que o usuário se torna sujeito ativo na produção da sua própria saúde, apesar de ser uma forma de cuidado transversal a qualquer cenário de atenção.

No percurso da análise, foi perceptível o desconhecimento dos enfermeiros em relação à PNPIC, em razão das lacunas no processo formativo e da falta de educação permanente, refletindo na falta de embasamento científico para atuar com essas práticas em seu cotidiano assistencial.

Neste estudo, foi possível também identificar as características das PICS, em que os profissionais pontuaram as possíveis terapêuticas que podem ser utilizadas no cuidado, percebendo que as mesmas possibilitam aumentar o vínculo entre profissional e usuário como também melhorar a qualidade da assistência na AB.

Outro achado pertinente foi a orientação de plantas medicinais e fitoterápicos, o que afeta positivamente no que diz respeito à diminuição da automedicação e a redução de gastos. Porém, percebeu-se certa confusão dos entrevistados na diferenciação dessas práticas, podendo levar a orientação incorreta, causando prejuízos ao usuário.

Vale ressaltar que os resultados obtidos, neste estudo, possuem limitações, uma vez que não se pode generalizar seus achados, em decorrência do fato de a investigação ter sido realizada em um município, que envolve culturas, saberes e crenças diferentes de outros locais do país. Além disso, aponta-se como dificuldade a insegurança e, em primeiro momento, a recusa inicial por parte de alguns enfermeiros em responder às perguntas norteadoras deste estudo, necessitando de maior diálogo e exposição da relevância, bem como repercussões positivas para a suas práticas, para que os mesmos pudessem participar da pesquisa. Apesar da boa adesão, ainda cinco enfermeiros se recursam a participar dessa investigação.

Por fim, vale salientar que esta pesquisa acrescenta novo olhar em relação às PICS, e traz contribuições para fins teórico-práticos da temática, considerando-o como novo instrumento de pesquisa, com o intuito de buscar melhorias na assistência dos enfermeiros no âmbito da $A B$, gerando, consequentemente, maior busca de outras formas de cuidado que não sejam focadas no curativismo, ampliando as concepções de saúde e cuidado da população.

Sugere-se que se desenvolvam mais estudos na área das PICS, tanto em outras regiões quanto também nos diversos âmbitos da saúde e com outros atores sociais. Visando a maior divulgação e credibilidade dessas práticas, ampliando sua implantação e implementação, com o intuito de transformar a presente realidade.

\section{REFERÊNCIAS}

1-Ministério da Saúde (BR). Secretaria de Atenção à Saúde. Política nacional de práticas integrativas e complementares no SUS. 2a ed. Brasília; 2015.

2-Ministério da Saúde (BR)- Portaria n ${ }^{\circ} 971$ de 03 de maio de 2006. Aprova a Política Nacional de Práticas Integrativas e Complementares (PNPIC) no Sistema Único de Saúde. Diário Oficial da União 2006.

3-Ministério da Saúde (BR). Portaria n 849 de 27 de março de 2017. Inclui a Arteterapia, Ayurveda, Biodança, Dança Circular, Meditação, Musicoterapia, Naturopatia, Osteopatia, Quiropraxia, Reflexoterapia, Reiki, Shantala, Terapia Comunitária Integrativa e Yoga à Política Nacional de Práticas Integrativas e Complementares. Diário Oficial da União 2017.

4-Ministério da Saúde (BR). Portaria no 702 de 21 de março de 2018. Altera a Portaria de Consolidação no 2/GM/MS, de 28 de setembro de 2017, para incluir novas práticas na Política Nacional de Práticas Integrativas e Complementares - PNPIC. Diário Oficial da União 2018.

5-Nascimento MVN, Oliveira I. As práticas integrativas e complementares grupais e sua inserção nos serviços de saúde da atenção básica. Estud Psicol. 2016;21(3):272-81. DOI: 10.5935/1678-4669.20160026

6-Rangel CT, Miranda FAN, Oliveira KKD. A terapia comunitária integrativa e a enfermagem: o fenômeno e seus contextos. Rev Pesqui Cuid Fundam. 2016;8(1):3770-9. DOI: 10.9789/21755361.2016.v8i1.3770-3779

7-Salles LF, Homo RFB, Silva MJP. Situação do ensino das práticas integrativas e complementares nos Cursos de Graduação em Enfermagem, Fisioterapia e Medicina. Cogitare Enferm. 2014;19(4): 741-6. DOI: 10.5380/ce.v19i4.35140

8-Lefèvre $F$, Lefèvre AMC. O discurso do sujeito coletivo: um novo enfoque em pesquisa qualitativa (desdobramentos). Caxias do Sul: Educs; 2005.

9-Oliveira AFP, Costa ICP, Andrade CG, Santos KFO, Anízio BKF, Brito FM. Fitoterapia na atenção básica: estudo com profissionais enfermeiros. Rev 
Pesqui Cuid Fundam. 2017;9(2): -480-7. DOI: 10.9789/2175-5361.2017.v9i2.480-487

10-Carvalho JLS, Nóbrega MPSS. Práticas integrativas e complementares como recurso de saúde mental na Atenção Básica. Rev Gaúcha Enferm. 2017;38(4):1-9. DOI: 10.1590/19831447.2017.04.2017-0014

11-Cruz PLB, Sampaio SF. As práticas terapêuticas não convencionais nos serviços de saúde: revisão integrativa. Rev APS 2016 [citado em $10 \mathrm{abr}$ 2017]; 19(3):483-94. Disponível em: https://aps.ufjf.emnuvens.com.br/aps/article/vie $\mathrm{w} / 2594 / 1054$

12-Silva ASP, Feitosa ST. Revisão sistemática evidencia baixo nível de conhecimento acerca da política nacional de práticas integrativas e complementares por parte de gestores e profissionais da saúde. Vitalle 2018;30(1):105-14. DOI: 10.14295/vittalle.v30i1.7491

13-Lopes ACP, Ceolin T, Ceolin S, Lope CV. As contribuições da disciplina "terapias complementares com ênfase em plantas medicinais" na prática profissional dos enfermeiros. Rev Pesqui Cuid Fundam. 2018; 10(3):619-25. DOI: 10.9789/21755361.2018.v10i3.619-625

14-Cinar N, Akduran F, Kose D. The attitudes of nursing students regarding the complementary and alternative medicine. Rev Eletr Enferm. 2016; 18:1-6. DOI: $10.5216 /$ ree.v18.37320

15-Caires JS, Andrade TA, Amaral JB, Calasans MTA, Rocha MDS. A utilização das terapias complementares nos cuidados paliativos: benefícios e finalidades. Cogitare Enferm. 2014; 19(3):514-20. DOI: 10.5380/ce.v19i3.33861

16-Honorio PG, Sosaya JF, Lázaro JZ, Alvarado GA, Obando VG, Valdera LW. Uso de terapias complementarias en el tratamiento de pacientes con lumbalgia crónica del seguro social de salud. la libertad, 2009. Rev Peruana Medicina Integrativa 2016;1(2):27-32. DOI: 10.26722/rpmi.2016.12.16

17-Rohr RV, Alvim NAT. Intervenções de enfermagem com música: revisão integrativa da literatura. Rev Pesqui Cuid Fundam. 2016;8(1):3832-44. DOI: $\quad \underline{10.1590 / 0104-}$ $\underline{070720160001020015}$

18-Costa CGA, Garcia MT, Ribeiro SM, Salandini MFS, Bógus CM. Hortas comunitárias como atividade promotora de saúde: uma experiência em Unidades Básicas de Saúde. Ciênc Saúde Coletiva 2015;20(10):3099-110. DOI: 10.9789/2175-5361.2016.v8i1.3832-3844
19-Azevedo ACB, Câmara ICP, Gois SRF, Benito LAO. Benefícios das Práticas Alternativas Integrativas e Complementares na Qualidade de Vida da Pessoa Idosa. Acta de Ciências e Saúde. 2015 [citado em 30 abr 2017]; 1(4):43-59. Disponível em: http://www2.ls.edu.br/actacs/index.php/ACTA/ar ticle/view/94/87

20-Batista LM, Valença AMG. A Fitoterapia no âmbito da atenção básica no SUS: realidades e perspectivas. Pesqui Bras Odontoped Clín Integr. 2012 [citado em 08 abr 2017]; 12(2):293-6. Disponível em: http://revista.uepb.edu.br/index.php/pboci/articl e/viewFile/1604/848

21-Ministério da Saúde (BR). Secretaria de Ciência, Tecnologia e Insumos Estratégicos. Programa nacional de plantas medicinais e fitoterápicos. Brasília: Ministério da Saúde; 2009. 22-Oliveira VB, Mezzomo TR, Moraes EF. conhecimento e uso de plantas medicinais por usuários de unidades básicas de saúde na Região de Colombo, PR. Rev Bras Cienc Saúde 2018; 22(1):57-64. DOI: 10.4034/RBCS.2018.22.01.08 23-Nascimento Júnior BJ, Tínel LO, Silva ES, Rodrigues LA, Freitas TON, Nunes XP et al. Avaliação do conhecimento e percepção dos profissionais da estratégia de saúde da família sobre o uso de plantas medicinais e fitoterapia em Petrolina-PE, Brasil. Rev Bras Plantas Med. 2016;18(1):57-66. DOI: $10.1590 / 1983-$ $\underline{084 X / 15 \quad 031}$

24-Neves RG, Pinho LB, Gonzáles RIC, Harter J, Schneider JF, Lacchini AJB. O conhecimento dos profissionais de saúde acerca do uso de terapias complementares no contexto da atenção básica. Rev Pesqui Cuid Fundam. 2012 [citado em 20 mar 2017]; 4(3):2502-9. Disponível em: http://www.redalyc.org/pdf/5057/505750894029 .pdf

25- Balouchi A, Mahmoudirad G, Hastings-Tolsma $M$, Shorofi AS, Shahdadi H, Abdollahimohammad A. Knowledge, attitude and use of complementary and alternative medicine among nurses: a systematic review. Complement Ther Clin Pract. 2018;31:146-57. DOI: 10.1016/i.ctcp.2018.02.008

Nota: Este estudo representa um recorte da Monografia de Conclusão de Curso intitulada "Práticas integrativas e complementares de saúde na atenção básica: análise de discurso dos enfermeiros" do Centro de Formação de Professores, da Universidade Federal de Campina Grande, como requisito para obtenção de título de Bacharel em Enfermagem. 
Recebido em: 29/01/2019

Aprovado em: 19/08/2019

Endereço de correspondência:

Marcelo Costa Fernandes

Rua Sérgio Moreira de Figueiredo $\mathrm{s} / \mathrm{n}$ - Casas Populares

CEP: 58900-000 - Cajazeiras/PB - Brasil

E- mail: celo cf@hotmail.com 